\title{
Computational modeling of neoclassical and resistive magnetohydrodynamic tearing modes in tokamaks
}

\author{
T. A. Gianakon, C. C. Hegna, and J. D. Callen \\ Department of Nuclear Engineering and Engineering Physics, 1500 Engineering Drive, Madison, \\ Wisconsin 53706-1687
}

(Received 2 July 1996; accepted 9 September 1996)

\begin{abstract}
Numerical studies of the nonlinear evolution of magnetohydrodynamic-type tearing modes in three-dimensional toroidal geometry with neoclassical effects are presented. The inclusion of neoclassical physics introduces an additional free-energy source for the nonlinear formation of magnetic islands through the effects of a bootstrap current in Ohm's law. The neoclassical tearing mode is demonstrated to be destabilized in plasmas which are otherwise $\Delta^{\prime}$ stable, albeit once an island width threshold is exceeded. The plasma pressure dynamics and neoclassical tearing growth is shown to be sensitive to the choice of the ratio of the parallel to perpendicular diffusivity $\left(\chi_{\|} / \chi_{\perp}\right)$. The study is completed with a demonstration and theoretical comparison of the threshold for single helicity neoclassical magnetohydrodynamic tearing modes, which is described based on parameter scans of the local pressure gradient, the ratio of perpendicular to parallel pressure diffusivities $\chi_{\perp} / \chi_{\|}$, and the magnitude of an initial seed magnetic perturbation. (C) 1996 American Institute of Physics. [S1070-664X(96)03212-0]
\end{abstract}

\section{INTRODUCTION}

The performance gains of the last several years in tokamak fusion plasmas has generated a resurgence in the observation of low helicity magnetic oscillations. ${ }^{1,2}$ Often, the onset of such oscillations either cause the plasma to disrupt violently, ${ }^{3,4}$ or the plasma confinement to degrade significantly. ${ }^{1}$ Experimental observations indicate that these instabilities are associated with magnetic reconnection-an interpretation based on the observation of the slow growth of these instabilities and mode numbers which are resonant in the plasma. Additionally, the electron cyclotron emission diagnostic indicates the presence of flatspots in the electron temperature profile about the resonant surface that are characteristic of magnetic island formation associated with the tearing events. ${ }^{1,2}$

One theoretical explanation for such modes is destabilization from the perturbed bootstrap current. Bootstrap currents arise from the viscous damping of the poloidal electron flow. The portion of the flow produced from the poloidal projection of the diamagnetic current when balanced against electron-ion friction yields a parallel current proportional to the cross-field pressure gradient, i.e., the bootstrap current. In the presence of a magnetic island, the pressure flattens within the island separatrix when parallel transport is fast relative to perpendicular transport. The pressure flattening eliminates the neoclassical bootstrap current within the magnetic island, but a cross-field pressure gradient remains outside the island separatrix. Since the pressure contours deform due to the island formation, a perturbed bootstrap current develops. For an equilibrium with $d p / d q<0$, where $p$ is the equilibrium pressure and $q$ is the inverse rotational transform, this perturbation produces a destabilizing effect. ${ }^{5-7}$

The destabilization mechanism is predicated on the assumption that the pressure equilibrates on the modified magnetic surfaces. When the island width is small enough, this is no longer a valid approximation as perpendicular transport mechanisms allow the pressure to cross magnetic surfaces faster than the pressure can equilibrate on the perturbed surfaces. When the magnetic island is smaller than a threshold value, the helical perturbation of the pressure profile about the island is insufficient to destabilize the island. An analytical model for the island dynamics which incorporates many of these features can be developed by using a nonlinear Rutherford theory ${ }^{8}$ amended to included neoclassical effects. ${ }^{9}$ In this particular model, the magnetic island width, $W$, is given by

$$
I_{1} \frac{d W}{d t}=\Delta^{\prime}+9.26 \epsilon_{s}^{0.5} \frac{\beta_{s}^{\prime}}{s_{s}} \frac{W}{W^{2}+W_{d}^{2}},
$$

where $I_{1} \simeq 0.8227, W_{d} \simeq 1.8 W_{c}, \Delta^{\prime}$ is the stability index from resistive magnetohydrodynamic (MHD) theory, ${ }^{10}$

$$
W_{c}=2.83\left(\frac{\chi_{\perp}}{\chi_{\|}}\right)^{0.25}\left(\frac{1}{\epsilon_{s} s_{s} n}\right)^{0.5},
$$

$\epsilon_{s}=\epsilon \rho_{s}, s_{s}=\rho_{s} q^{\prime} q^{-1}, \beta_{s}^{\prime}=-\beta_{0} q_{s}^{2} \epsilon_{s}^{-2} p_{s}^{\prime}$. Here, $\chi_{\perp}$ and $\chi_{\|}$are the perpendicular and parallel pressure diffusivities, respectively, the mode is resonant at $\rho \equiv r / a=\rho_{s} \equiv r_{s} / a$ where $q=m / n, p_{s}^{\prime}$ is the pressure gradient evaluated at the resonant surface normalized to the pressure on-axis, $\Delta^{\prime}$ is the tearing mode matching parameter, and $\beta_{0}$ is the normalized pressure on axis. (Note that the introduction of $\beta_{0}$ is strictly for convenience when making comparisons with the later numerical simulations; the real dependency is on the unnormalized local pressure gradient.) The last term in Eq. (1) describes the perturbed bootstrap current which is typically destabilizing in tokamaks. The novel aspect of neoclassical instabilities is that magnetic islands can occur even in the limit of resistive MHD tearing stability, $\Delta^{\prime}<0$. This model is essentially the equivalent of the $\mathrm{Qu}$ and Callen model ${ }^{5}$ or the model of Carrera et al. ${ }^{6}$ in the limit $\chi_{\|} / \chi_{\perp} \rightarrow \infty$, i.e., where the pressure completely equilibrates on each flux surface.

The dynamics of the island evolution model can be summarized in the simple phase space diagram of Figure 1 which 


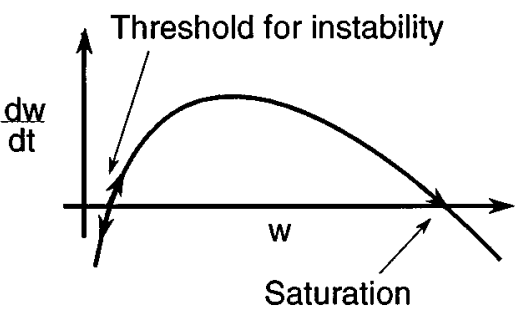

FIG. 1. Phase diagram of the neoclassical MHD tearing mode.

assumes $\Delta^{\prime}<0$. The diagram shows two fixed points: a nonlinear island threshold, below which island formation is suppressed, and a large saturated island width. The two fixed points can be determined by setting the growth rate of Eq. (1) to zero and solving the resultant quadratic for the island width. For a sufficiently well separated threshold and saturation width, the solution to the quadratic approximates as

$$
\begin{aligned}
& W_{\text {threshold }} \simeq \frac{2.80}{n} \frac{\left|\Delta^{\prime}\right|}{\beta_{s}^{\prime} \epsilon_{s}^{1.5}}\left(\frac{\chi_{\perp}}{\chi_{\|}}\right)^{0.5}, \\
& W_{\text {sat }} \simeq 9.26 \epsilon_{s}^{0.5} \frac{\beta_{s}^{\prime}}{\left|\Delta^{\prime}\right| s_{s}} .
\end{aligned}
$$

At small island width the perpendicular pressure transport dominates over the parallel transport and the pressure profile is unaffected by the island structure, which then produces no perturbed bootstrap current, and no island growth. As the island width is increased, the island eventually perturbs the pressure profile and the perturbed bootstrap current is then able to destabilize the island. An increase in the plasma pressure gradient shifts the entire phase space curve upward. This then reduces the threshold for the mode and also increases the saturated island width. Alternatively, an increase in the parallel pressure diffusivity primarily shifts the instability threshold to smaller amplitude, but leaves the saturated state at nearly the same value. This simple neoclassical MHD model has shown remarkable dynamical agreement with the experimental observations on the Tokamak Fusion Test Reactor (TFTR). $1,2,11$

To further explore the dynamics of the neoclassical MHD tearing mode in a realistic toroidal geometry, numerical simulations based on neoclassical reduced MHD equations have been conducted. In Section II, the model equations for neoclassical reduced MHD as implemented in the neofar code are presented. In Section III, the dynamics of pressure equilibration on magnetic field lines in the presence of an anisotropic diffusivity is presented. In Section IV, simulation results based on this neoclassical MHD model are presented. The results are summarized in Section V.

\section{MODEL EQUATIONS}

In this section, a set of equations for the numerical simulation of resistive instabilities in a tokamak are presented which include neoclassical viscous stress effects. The derivation of this reduced set of equations is presented elsewhere. ${ }^{12}$ The model is formulated based on a geometry defined by an ideal MHD equilibria in an axisymmetric toroidal geometry. This equilibrium is solved numerically with the fixed boundary, equilibrium, Grad-Shafranov code RSTEQ ${ }^{13-15}$ and is used to define a nonorthogonal "straight-field-line", coordinate system with the associated covariant and contravariant metric elements. The full set of two fluid equations are not solved; instead, after a decomposition of variables into equilibrium (subscript 0) and fluctuation components (subscript 1), a set of neoclassical reduced MHD evolution equations for the poloidal flux, the toroidal vorticity, and the plasma pressure are used that are valid in the limit of small inverse aspect ratio $(\epsilon \ll 1)$ and low plasma pressure $(\beta \ll 1)$. This decomposition into fluctuation and equilibrium quantities also retains the coupling of poloidal harmonics due to toroidicity, since the details of the equilibrium quantities are not expanded in any small parameter. The equations are implemented and solved numerically in the code neofar which is based on the formalism of the initial value code FAR. ${ }^{16}$

In axisymmetric systems the equilibrium magnetic field may be expressed as

$$
\vec{B}=I \nabla \zeta+\nabla \zeta \times \nabla \psi_{0},
$$

where $I$ can be shown to be a flux function from axisymmetry. When the radial projection of the ideal MHD equilibrium force balance is combined with Ampère's law and the representation of the magnetic field, the result is the GradShafranov equation, ${ }^{17}$ which is expressed as

$$
R^{2} \nabla \cdot\left(\frac{1}{R^{2}} \nabla \psi_{0}\right)=-\frac{\beta_{0}}{2 \epsilon^{2}} R^{2} \frac{d p}{d \psi_{0}}-\frac{1}{\epsilon^{2}} I \frac{d I}{d \psi_{0}},
$$

where the following normalizations have been made. ${ }^{14}$ Length scales have been normalized by the plasma minor radius $a=\epsilon R_{0}$, where $R_{0}$ is the Shafranov-shifted major axis and $\epsilon$ is the inverse aspect ratio defined as

$$
\epsilon^{2}=\frac{1}{2 \pi^{2}} \int_{V} d^{3} V \frac{1}{R^{2}},
$$

but with the exception that the cylindrical coordinate $R$ has been normalized by $R_{0}$ and $d^{3} V$ has been normalized by $R_{0}^{3}$. The magnetic field has been normalized by the value (in units of Gauss) $B^{*}=I_{\text {wall }} R_{0}^{-1}$, where $I / I_{\text {wall }}=1$ at the plasma edge. The flux $\psi_{0}$ has been normalized by $\psi^{*}=-\epsilon^{2} R_{0} I_{\text {wall }}$. The pressure is normalized to $p_{0}$, the pressure on axis, which leads to the definition $\beta_{0}=8 \pi p_{0} R_{0}^{2} / I_{\text {wall }}^{2}$. In the simulations which follow, $R_{0}=2.45 \mathrm{~m}$ and $\epsilon \simeq 0.33$. Also, a new equilibrium flux coordinate $\rho$ is introduced which is defined by $d \psi_{0} / d \rho=\rho I / q$, with normalization that $\rho=0$ at the equilibrium magnetic axis and $\rho=1$ at the conducting wall.

At this point it is also convenient to define two timescales which will be relevant for the initial value equations. The first is the resistive time $\tau_{R}$ which is defined as $\tau_{R}=\epsilon^{2} R_{0}^{2} \eta^{-1} 4 \pi c^{-2}$, and will be used to normalize all times. The second is the Alfvén time $\tau_{A}$ which is defined as $\tau_{A}^{-2}=I_{\text {wall }}^{2} \rho_{D}^{-1} R_{0}^{-4}(4 \pi)^{-1}$. The ratio of these two timescales, $S=\tau_{R} / \tau_{A}$, is defined as the Lundquist or magnetic Reynolds number and for a hot tokamak plasma has the property $S \gg 1$; e.g., in TFTR $S \sim 10^{8}$. 
The model presented here is a close derivative of the reduced-MHD models of both Strauss ${ }^{18}$ and also Hazeltine and Meiss, ${ }^{19}$ but while these models neglect viscous stress tensor effects, this model includes such effects based on neoclassical closure arguments. ${ }^{20}$ This neoclassical reduced MHD model consists of a lowest order in $\epsilon$ parallel projection of Ohm's law, which is given by

$$
\begin{aligned}
\frac{\partial \psi_{1}}{\partial \tau}= & \frac{I}{R^{2}}\left[\frac{\partial \phi_{1}}{\partial \zeta}-\frac{1}{q} \frac{\partial \phi_{1}}{\partial \theta}\right]-\frac{1}{\rho R^{2}} \frac{\partial \phi_{1}}{\partial \theta} \frac{\partial \psi_{1}}{\partial \rho} \\
& +\frac{1}{\rho R^{2}} \frac{\partial \phi_{1}}{\partial \rho} \frac{\partial \psi}{\partial \theta}+R^{2} J_{1}^{\zeta}-\frac{R^{2}}{I} \vec{B} \cdot \nabla \cdot \overrightarrow{\vec{\pi}},
\end{aligned}
$$

where the toroidal projection of the current is

$$
J_{1}^{\zeta}=\nabla \zeta \cdot \vec{J}_{1}^{\zeta}=\nabla \cdot \frac{1}{R^{2}} \nabla \psi_{1}-\frac{1}{R^{4}} \frac{\partial^{2} \psi_{1}}{\partial \zeta^{2}}
$$

and the parallel projection of the stress-tensor term is defined in the Appendix.

The second evolution equation is for the toroidal projection of the vorticity $w_{1}^{\zeta}$, which is given by

$$
\begin{aligned}
\frac{\partial w^{\zeta}}{\partial \tau}= & \frac{1}{\rho R^{2}} \frac{\partial \phi_{1}}{\partial \rho} \frac{\partial w^{\zeta}}{\partial \theta}-\frac{1}{\rho R^{2}} \frac{\partial \phi_{1}}{\partial \theta} \frac{\partial w^{\zeta}}{\partial \rho}+S^{2}\left\{\frac{1}{\rho R^{2}} \frac{\partial \psi_{1}}{\partial \theta} \frac{\partial J_{0}^{\zeta}}{\partial \rho}-\frac{1}{\rho R^{2}} \frac{\partial \psi_{1}}{\partial \rho} \frac{\partial J_{0}^{\zeta}}{\partial \theta}-J_{1}^{\rho} \frac{\partial}{\partial \rho}\left(\frac{I}{R^{2}}\right)-J_{1}^{\theta} \frac{\partial}{\partial \theta}\left(\frac{I}{R^{2}}\right)\right. \\
& \left.+\frac{I \rho}{R^{2} q} \frac{1}{\rho} \frac{\partial}{\partial \theta} J_{1}^{\zeta}+\frac{I}{R^{2}} \frac{\partial}{\partial \zeta} J_{1}^{\zeta}+\frac{1}{\rho R^{2}} \frac{\partial \psi_{1}}{\partial \theta} \frac{\partial J_{1}^{\zeta}}{\partial \rho}-\frac{1}{\rho R^{2}} \frac{\partial \psi_{1}}{\partial \rho} \frac{\partial J_{1}^{\zeta}}{\partial \theta}\right\}-\nabla \zeta \cdot \nabla \times \nabla \cdot \overrightarrow{\vec{\pi}},
\end{aligned}
$$

where $w_{1}^{\zeta}$ is given by Eq. (9) with $\psi_{1}$ replaced by $\phi_{1}$.

The interpretation of each of these terms is as follows. The first two terms on the right-hand-side represent the convection of vorticity; the $\vec{J}_{1}^{\rho}$ and $\vec{J}_{1}^{\theta}$ are curvature pieces and expand as

$$
\begin{aligned}
J_{1}^{\rho} \frac{\partial}{\partial \rho}\left(\frac{I}{R^{2}}\right)+J_{1}^{\theta} \frac{\partial}{\partial \theta}\left(\frac{I}{R^{2}}\right)= & \left\{\frac{1}{\rho} \frac{\partial^{2} \psi_{1}}{\partial \theta \partial \zeta}\right\}\left\{\frac{\hat{g}^{\rho \theta}}{R^{2}} \frac{\partial}{\partial \rho}\left(\frac{I}{R^{2}}\right)+\frac{\hat{g}^{\theta \theta}}{R^{2}} \frac{1}{\rho} \frac{\partial}{\partial \theta}\left(\frac{I}{R^{2}}\right)\right\}+\left\{\frac{1}{\rho} \frac{\partial^{2} \psi_{1}}{\partial \rho \partial \zeta}\right\}\left\{\frac{\hat{g}^{\rho \rho}}{R^{2}} \frac{\partial}{\partial \rho}\left(\frac{I}{R^{2}}\right)+\frac{\hat{g}^{\rho \theta}}{R^{2}} \frac{1}{\rho} \frac{\partial}{\partial \theta}\left(\frac{I}{R^{2}}\right)\right\} \\
& +\left\{\frac{1}{\rho} \frac{\partial p_{1}}{\partial \theta}\right\}\left\{\frac{-\beta_{0}}{\epsilon^{2}} \frac{1}{I} \frac{\partial}{\partial \rho}\left(\frac{I}{R^{2}}\right)\right\}+\left\{\frac{\partial p_{1}}{\partial \rho}\right\}\left\{\frac{\beta_{0}}{\epsilon^{2}} \frac{1}{I} \frac{1}{\rho} \frac{\partial}{\partial \theta}\left(\frac{I}{R^{2}}\right)\right\} .
\end{aligned}
$$

The last two terms of Eq. (11) are often referred to as the pressure curvature pieces and lead to the Glasser effect, ${ }^{21,22}$ which produces a stabilizing effect linearly and nonlinearly when the equilibrium has good average curvature. However, the bootstrap current dominates over these terms and so they have been dropped in the following simulations. The 6 remaining current pieces are the linear and nonlinear portions of $\vec{B} \cdot \nabla J^{\zeta}$. The final stress tensor term is the ion viscosity which is composed of a Braginskii-like term given as $\nu \Delta^{*} w^{\zeta}$ and also a neoclassical term described in the Appendix. The final evolution equation is for the perturbed pressure, $p_{1}$, which is given by

$$
\frac{\partial p_{1}}{\partial t}+\vec{v}_{1} \cdot \nabla p_{0}+\vec{v}_{1} \cdot \nabla p_{1}=-\nabla \cdot \vec{q}_{1},
$$

where the equilibrium heat source has been balanced with the equilibrium portion of the heat flux $\left(Q=-\chi_{\perp} \nabla^{2} p_{0}\right)$ and changes to the resistive heating are deemed to be small. The $\vec{q}_{1}$ fluctuation is given by

$$
\vec{q}_{1}=-\chi_{\perp} \nabla p_{1}-\left(\chi_{\|}-\chi_{\perp}\right)\left[\frac{\vec{B} \vec{B} \cdot \nabla p_{1}}{B^{2}}+\frac{\vec{B} \vec{B}_{1} \cdot \nabla p_{0}}{B^{2}}\right],
$$

where $\vec{B}_{0} \cdot \nabla p_{0}$ is equal to zero, since the equilibrium pressure profile is a flux function [i.e., $p_{0}=p_{0}(\rho)$ and $\vec{B} \cdot \nabla \rho=0]$. Also, the diffusivity has been normalized by $\epsilon^{2} R_{0}^{2} / \tau_{R}$ and factors of $1 / B^{2} \simeq 1 / B_{0}^{2}$.
The numerical model implemented in the neofar code is equivalent to that of its parent code FAR which is an initial value code that solves the linear portion implicitly with a block tridiagonal solve and the nonlinear term explicitly. The explicit portion (and especially that nonlinear portion associated with the parallel pressure transport) generates the strongest constraints on the numerical implementatation due to stability requirements.

While neofar has the potential to handle the poloidal mode coupling associated with toroidicity, in the simulations which follow, only a single harmonic $(m / n=2 / 1,3 / 1$, or $3 / 2)$ is considered in addition to the equilibrium harmonics $(\mathrm{m} / \mathrm{n}$ $=0 / 0$ through $10 / 0$ ).

\section{PRESSURE EVOLUTION DYNAMICS}

The model in this paper uses an anisotropic pressure diffusivity which accounts for differences in the perpendicular $\left(\chi_{\perp}\right)$ and parallel $\left(\chi_{\|}\right)$heat transport. In a real plasma these coefficients, along with the plasma resistivity, would depend on various plasma parameters (temperature, density, turbulence levels, etc.). However, the assumption has been made that in the vicinity of any particular island the effect of topological changes associated with the island are likely to be dominant over these spatial variations. Consequently, $\chi_{\perp}$ and $\chi_{\|}$will be assumed to be constants across the entire extent of the plasma. In principle, neofar could be run in a 
fully nonlinear turbulent mode with dynamic versions of $\chi_{\perp}$ and $\chi_{\|}$which could address such effects, but at the expense of more complicated nonlinear terms and much longer computing times.

The particular choice of values for $\chi_{\perp}$ and $\chi_{\|}$will reflect three timescales: the parallel equilibration time, the energy confinement time, and the resistive time. The ratio of the first two timescales is based on the observation that free streaming of electrons along the magnetic field lines produces rapid equilibration of pressure along a field line, while the pressure transport across the magnetic field lines is constrained. This implies that $\chi_{\|} / \chi_{\perp} \gg 1$. This choice of $\chi_{\|}$also plays a significant role in the determination of a short enough time step to avoid numerical instability associated with the explicit time advance of the nonlinear portion of the parallel heat flux, yet long enough to simulate the island growth over a fraction of the resistive timescale. The ratio of the last two timescales is based on the experimental observation that the energy confinement time $(\sim 100 \mathrm{~ms}$ in TFTR $)$ is faster than the resistive timescale $(\sim 10 \mathrm{~s}$ in TFTR), which implies a dimensionless $\chi_{\perp} \sim 100$. This choice of $\chi_{\perp}$ is central to ensuring that the pressure "rapidly" equilibrates while the island evolves, rather than the pressure gradients external to the magnetic island steepening during island growth or significantly lagging the evolution of the magnetics, i.e., the pressure relaxation has not completely propagated to the magnetic axis but requires a finite time of order of the energy confinement time to equilibrate throughout the plasma. The general criterion required to avoid pressure steepening is $\chi_{\perp}^{-1} W d W / d t \ll 1 .^{23}$ For $\Delta^{\prime}$ unstable modes this can sometimes be violated during the early linear evolution of the mode, but since this is associated with an extremely small magnetic island, this regime is largely irrelevant for experimental comparisons and will not be an issue for the choice of parameters used for the bootstrap current driven modes of this paper.

For $\chi_{\perp}=100$ and $\chi_{\|}=10^{7}$, the pressure dynamics can be described based on rapid parallel transport which effectively "short-circuits" the perpendicular pressure gradient across the island region. This flattening of the pressure profile across the island appears as a pressure decrease in the $0 / 0$ harmonic of the pressure between the magnetic axis and the island. A simple estimate of this pressure drop on axis based on the "belt" model of Chang and Callen ${ }^{24}$ is $p_{0 / 0}=-W d p_{0} /\left.d \rho\right|_{\rho_{0}}$, where $W$ is the island width and $d p_{0} / d \rho$ is the equilibrium pressure gradient evaluated at the rational surface.

To test the robustness of the numerical simulations, to assess the helical structure of the pressure contours, and to assess the pressure equilibration on the magnetic flux surfaces-a feature which is necessary for the threshold destabilization of the neoclassical tearing mode - a single large "static" magnetic island is considered but in the absence of a pressure convection term. In an ideal situation, where $\chi_{\|} / \chi_{\perp} \rightarrow \infty$ or where the magnetic fluctuations are zero, the pressure contours and field lines would exactly coincide. However, in the vicinity of the island X-point even though the perpendicular transport is slow, the transit time across the $\mathrm{X}$-point for a small distance $\delta_{x}$ is quite short: $\tau_{\perp}=k_{\perp}^{2} \chi_{\perp} \simeq \chi_{\perp} /\left(\delta_{x}\right)^{2}$. On the other hand, the singular na-

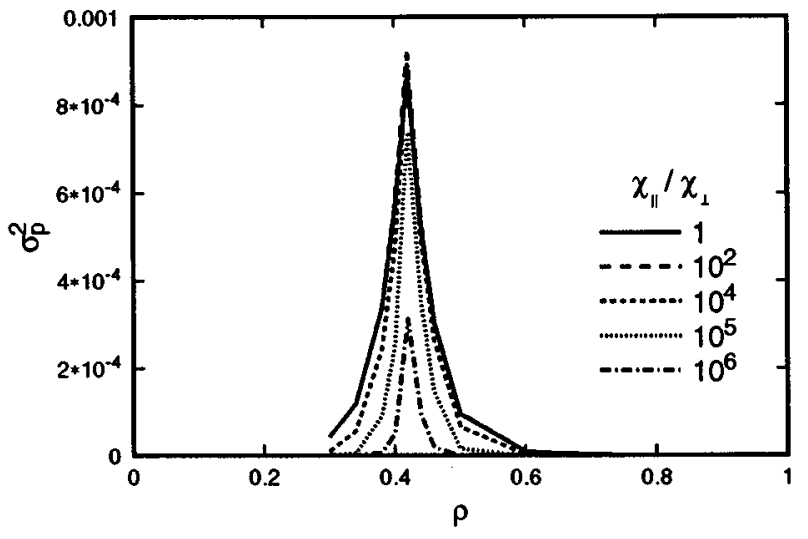

FIG. 2. An increase in $\chi_{\|} / \chi_{\perp}$ increases the degree to which the plasma pressure equilibrates on the flux surface. The pressure variance is computed by following individual field lines approximately 3200 times around the torus (200000 iterates at a $\Delta \zeta=0.1$ step size) and computing the pressure along the field line. This pressure is used to compute the mean pressure on a field line and also the pressure variance. Initial starting points cross through an island $\mathrm{X}$-point.

ture of the separatrix requires heat to travel an infinite distance in the parallel direction to cross the X-point: $\tau_{\|}=k_{\|}^{2} \chi_{\|}$. If a Taylor expansion of a mode is made about the rational surface, then $k_{\|}=\delta_{x}(m / q)(d q / d \rho)$. A balance between these two timescales generates a scale length at which the transport changes form being parallel to perpendicular dominated. The scale length is

$$
\left.\delta_{x} \simeq\left(\frac{\chi_{\perp}}{\chi_{\|}}\right)^{0.25}\left(\frac{m}{q} \frac{d q}{d \rho}\right)^{-0.5}\right|_{\rho=\rho_{0}},
$$

which for realistic plasmas is very small $\left(\sim 10^{-3}\right)$. This combination of scale lengths and timescales allows the island to support a weak pressure gradient across the $\mathrm{X}$-point rather than producing a distinct $\mathrm{X}$-point in the pressure contours. This feature proves to be pivotal for the amplitude threshold for generation of the neoclassical MHD instabilities of the next section.

The degree to which the pressure has equilibrated along a flux surface can be quantified by computing the pressure variance $\left(\sigma^{2}\right)$ along any particular magnetic field line. In Figure 2, this pressure variation is presented for simulations based on a static magnetic island of approximately $20 \%$ of the minor radius for various levels of $\chi_{\|} / \chi_{\perp}$ with select starting points which cross in the vicinity of an island $\mathrm{X}$-point. This variance should be contrasted with a diagnostic-like variance which would sample pressure from different magnetic field-lines but at a constant radius. As expected, the pressure variation is largest near the resonant rational magnetic surface, and for $\chi_{\|} / \chi_{\perp}=10^{6}$ has a magnitude of $\sim 1-2 \%$. The variance is largest near the mode rational surface, because the field line passes through the vicinity of the island X-point where transport is dominated by perpendicular diffusion to regions near the separatrix across the island O-point where transport is dominated by parallel dif- 


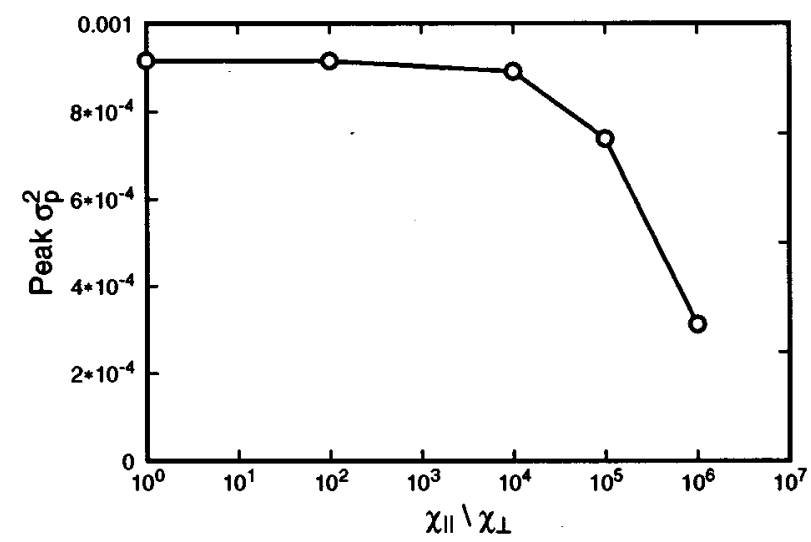

FIG. 3. An increase in $\chi_{\|} / \chi_{\perp}$ decreases the peak pressure variance. The peak is located at or near the mode rational surface.

fusion. This trend is further clarified in Figure 3 which illustrates that the peak pressure variance decreases as a function of $\chi_{\|} / \chi_{\perp}$.

The final issue which the pressure evolution affects is the numerical stability of the time advance scheme, especially due to the explicit nature of the nonlinear terms. A reasonable choice of timestep during the nonlinear phase of mode growth to ensure stability for a value of $\chi_{\|}=10^{7}$ is $10^{-9} \tau_{R}$ when $\psi_{1} \sim 10^{-4}$. As $\psi_{1}$ increases in value this timestep must be further reduced to ensure stability. With this timestep and with the advantage of larger timesteps in the early linear phase of island growth, a typical run of 15 modes requires roughly 2 weeks in real time on an IBM/ RISC6000 model 370. This necessity of balancing compute times with numerical stability requirements will limit the simulations to unrealistically small values of $\chi_{\|} / \chi_{\perp}$. While this solution will not generate the pressure equilibration equivalent to experimental conditions, it will be sufficient to elucidate the threshold destabilization of the neoclassical MHD tearing mode.

\section{NEOCLASSICAL TEARING MODES}

Neoclassical effects arise from the viscous damping of the poloidal electron flow. The portion of the flow produced from the poloidal projection of the diamagnetic current when balanced against electron-ion friction yields a parallel current proportional to the cross-field pressure gradient, the bootstrap current. When $\left(d p_{0} / d \rho\right) /(d q / d \rho)<0$, this perturbation reinforces the magnetic perturbation of the island and an instability may grow in time. [The neoclassical modes are predicted to be stable in reversed shear tokamak discharges where $\left(d p_{0} / d \rho\right) /(d q / d \rho)>0$.]

In the limit $\chi_{\|} / \chi_{\perp} \rightarrow \infty$, the pressure exactly equilibrates on the magnetic flux surfaces $(\vec{B} \cdot \nabla p=0)$, and the magnetic island grows from very small amplitude. However, the introduction of finite values of $\chi_{\|}$and $\chi_{\perp}$ at small island amplitude produce an insufficient perturbation of the pressure profile to destabilize the neoclassical magnetic island-a threshold in magnetic island width, $W_{\text {threshold }}$. As described in Section I, when the island width is smaller than this width

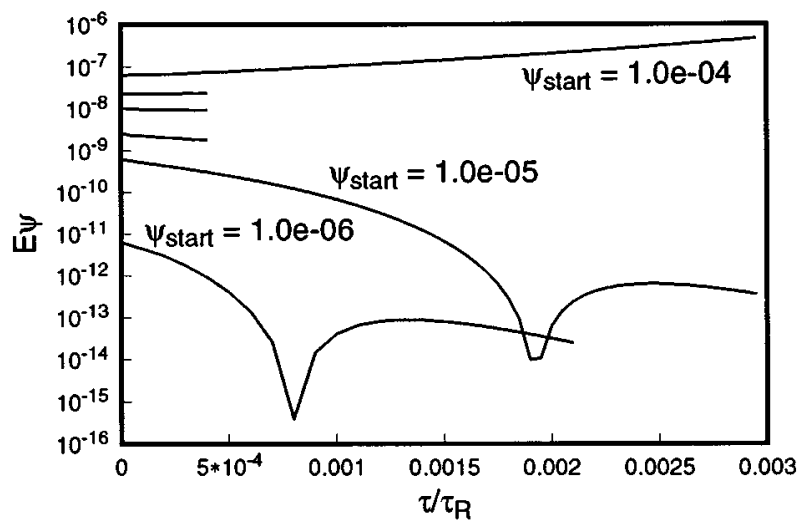

FIG. 4. Destabilization of the neoclassical tearing mode requires a finite perturbation to initiate the mode. Here, $\Psi_{\text {start }}$ is the magnitude of the initial perturbation evaluated at the mode rational surface. The initial profile shape decays toward zero value at both boundaries. The oscillatory behavior of the decaying solutions is a linear effect driven by $\vec{B} \cdot \nabla \phi_{1}$ causing $\psi_{1}$ to oscillate as it decays.

$\left[W<W_{\text {threshold }}\right.$, where $W_{\text {threshold }}$ is given by Eq. (4)], the island width decays in amplitude; however when the island is larger than this width, ( $\left.W>W_{\text {threshold }}\right)$ the island width grows in amplitude. This dependence on the initial perturbation magnitude is illustrated in Figure 4 for an equilibrium $q$-profile of $q=1.01\left(1+\left(2.2 \psi_{0}\right)^{4}\right)^{1 / 2}$, a $p$-profile of $p_{0}=\left(1-\psi_{0}^{2}\right)$ with $\beta_{0}=0.068$, and where $\psi_{1}$ is initialized to

$$
\begin{aligned}
& \psi_{1, m / n}(t=0)=\Psi_{\text {start }}\left(\rho / \rho_{s}\right)^{m} ; \quad \rho<\rho_{s}, \\
& \psi_{1, m / n}(t=0)=\Psi_{\text {start }}\left(1-\rho^{-m}\right) /\left(1-\rho_{s}^{-m}\right) ; \quad \rho>\rho_{s},
\end{aligned}
$$

where $\Psi_{\text {start }}$ is the value of $\psi_{1}$ at the resonant surface, $\rho_{s}$.

The neoclassical MHD threshold is also strongly dependent on the local pressure gradient as parameterized by $\beta_{0}$ since the bootstrap current depends directly on the gradient of the plasma pressure. Subsequently, as the pressure gradient is increased, a given initial seed magnetic perturbation may cross the threshold and be destabilized, as is illustrated in Figure 5.

Much of the prior analysis can be combined into a threshold curve for a given $\chi_{\|} / \chi_{\perp}$ by performing parameter

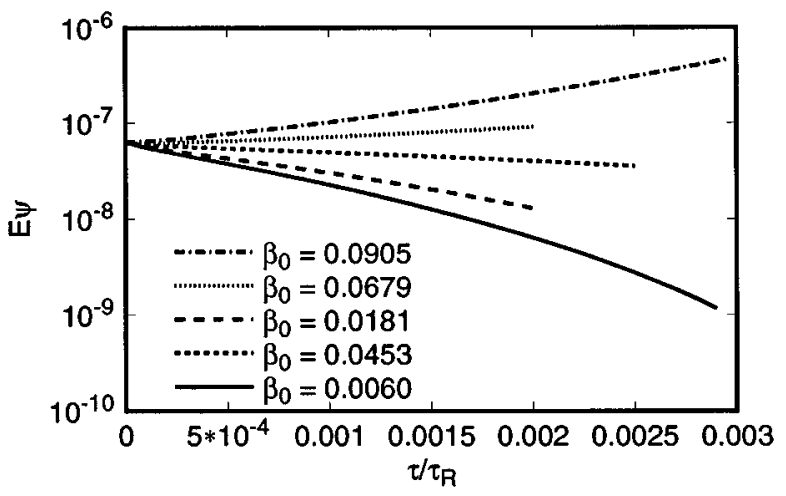

FIG. 5. For a given initial magnetic perturbation, an increase in plasma pressure causes destabilization of the neoclassical MHD tearing mode. 


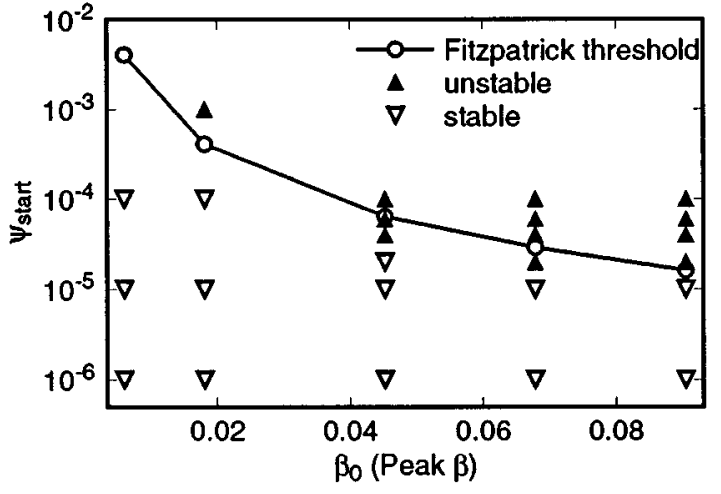

FIG. 6. Neoclassical MHD tearing threshold for the $2 / 1$ mode based on numerical simulations are in rough agreement with the predictions of neoclassical MHD theory.

scans over $\beta_{0}$ and $\Psi_{\text {start }}$. Figures 6,7 , and 8 provide such a threshold curve for the $2 / 1,3 / 1$, and $3 / 2$ modes, respectively, and compare the numerically computed threshold with the analytic predictions of the threshold. In general, the numerical simulations show good qualitative agreement with the theoretical predictions and confirm that as the pressure gradient is increased, a successively smaller seed perturbation is required for destabilization of the neoclassical MHD tearing mode. These figures also illustrate a quantitative difference between the theory and the simulations. This difference is not a real deficiency in the model, but rather due to inaccuracies in determining the appropriate value of $\Delta^{\prime}$ to be used in the theoretical prediction. The simulations do not compute a value of $\Delta^{\prime}$. However in the absence of the bootstrap current, at no level of perturbation is a single helicity tearing mode found to be unstable - a strong indication that $\Delta^{\prime}<0$. Instead, the assumption has been made that $\Delta^{\prime}=-2 \mathrm{~m} / \rho_{\mathrm{s}}$. However, any negative value of $\Delta^{\prime}$ would have sufficed to generate a similar threshold curve. [If $\rho$ were a cylindrical coordinate, then $q(\rho)$ generates the following values for the cylindrical $\Delta^{\prime}: \quad \rho_{s} \Delta_{2 / 1}^{\prime}=-0.22, \quad \rho_{s} \Delta_{3 / 1}^{\prime}=-5.4, \quad$ and $\left.\rho_{s} \Delta_{3 / 2}^{\prime}=-5.7.\right]$

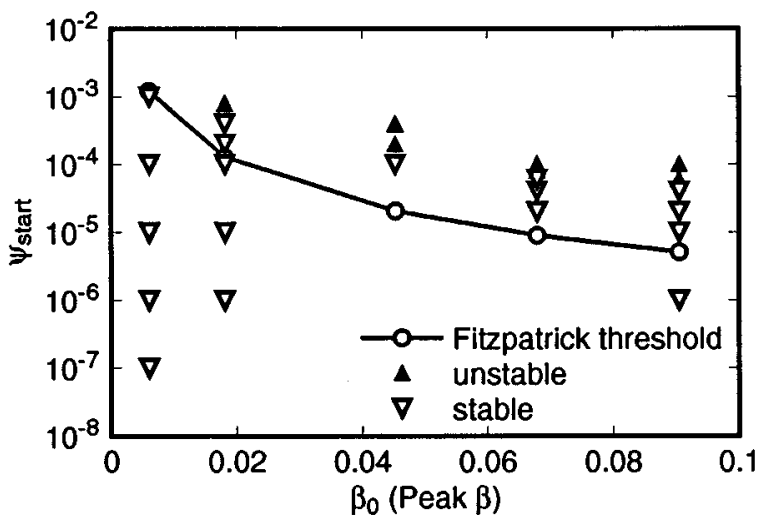

FIG. 7. Neoclassical MHD tearing threshold for the $3 / 1$ mode based on numerical simulations are approximately a factor of 10 larger than the predictions of neoclassical MHD theory.

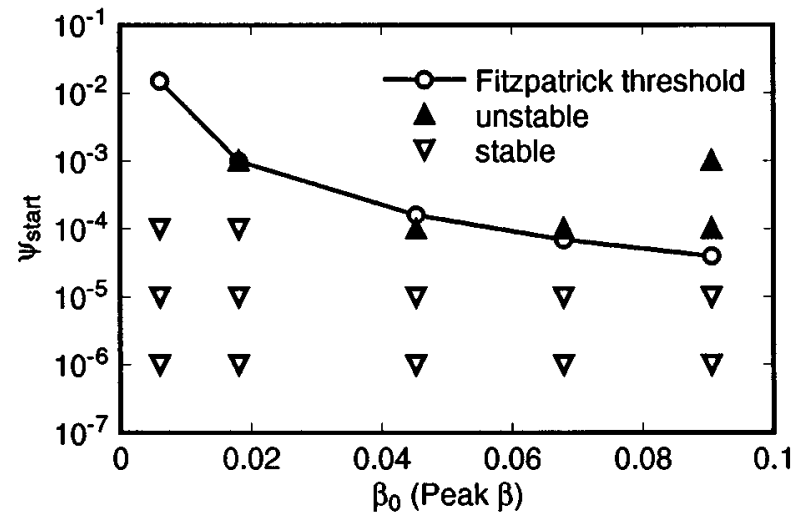

FIG. 8. Neoclassical MHD tearing threshold for the $3 / 2$ mode based on numerical simulations are approximately the same as the predictions of neoclassical MHD theory.

The most important feature which can lower the neoclassical tearing mode threshold is an increase in the ratio of $\chi_{\|} / \chi_{\perp}$. Unfortunately, numerical difficulties preclude increasing this ratio to a TFTR relevant value of $\chi_{\|} / \chi_{\perp} \sim 10^{10}$. However, decreasing this ratio as is illustrated in Figure 9 demonstrates the strong stabilizing/destabilizing role this ratio plays, since previously unstable initial conditions have now been stabilized.

Once the island is over the neoclassical threshold and the initial transients have decayed away, the island width growth rate is proportional to the island width as illustrated in Figure 10. In this particular case, the island width has reached over $20 \%$ of the plasma minor radius. However, the calculation has been terminated at this point because in order to maintain the stability of the pressure evolution the time step must be reduced below $0.01 \tau_{A}$, which would then imply much longer computing times. Also, the predicted saturation level for the mode is beyond the size of the tokamak, so saturation is not likely to be obtained. In either case, the small island assumption of the analytic theory is also violated and the theory cannot be accurately applied to explain the simulation. Neither the large thresholds nor the large saturation

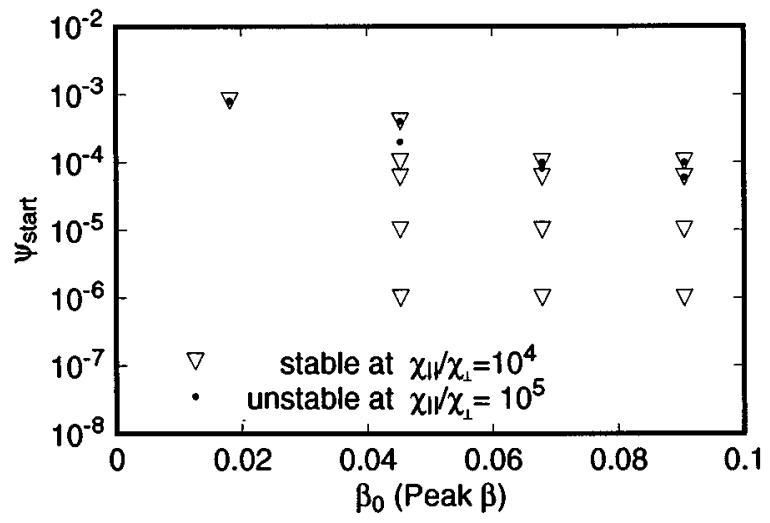

FIG. 9. Reduction of $\chi_{\|} / \chi_{\perp}$ to $10^{4}$ increases the neoclassical threshold. Initial conditions which were unstable at $\chi_{\|} / \chi_{\perp}=10^{5}$ are now stable. 


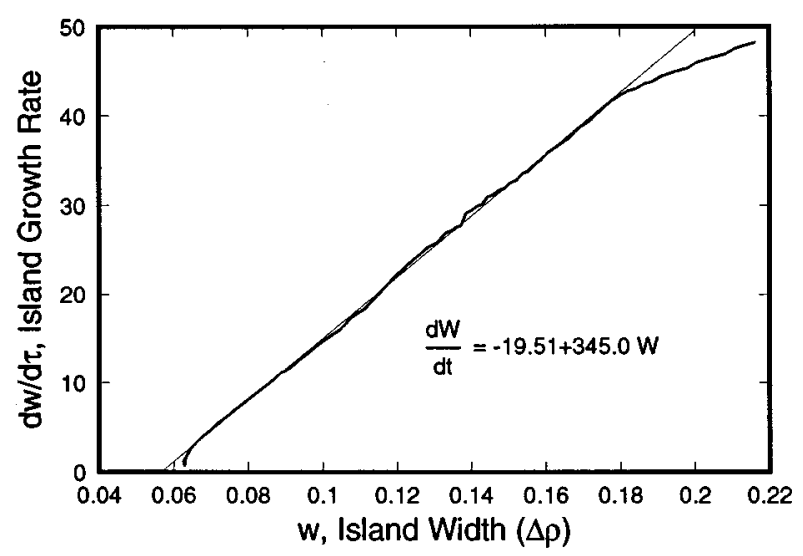

FIG. 10. After the neoclassical threshold, the island width grow rate is proportional to the island width, until nonlinear effects begin to slow the island growth. The simulation was terminated at an island width of $20 \%$ of the minor radius due to numerical problems.

levels presented in the prior simulations should be construed as a generic part of neoclassical MHD. In order to simulate the physics which has been described, rather extreme parameter ranges have been required which are in general not realizable in actual machines. However, this was required since the simulations are limited by $\chi_{\perp} / \chi_{\|}$.

\section{SUMMARY}

The reduced magnetohydrodynamics (MHD) paradigm has been extended to include viscous force $(\nabla \cdot \overrightarrow{\vec{\pi}})$ effects based on neoclassical closures. This neoclassical reduced MHD model consists of evolution equations for the perturbed flux, toroidal vorticity, and plasma pressure, through a parallel Ohm's Law, a toroidal vorticity evolution, and a plasma pressure evolution equation. The plasma pressure evolution includes an anisotropic pressure diffusivity to account for rapid parallel energy transport. The viscous stress tensor adds a bootstrap current contribution to the parallel Ohm's law [Eq. (8)] and a neoclassical viscosity to the vorticity evolution equation [Eq. (10)]. The model is principally valid only in the large aspect ratio limit due to the assumptions of plasma incompressibility and the use of only a single stream function to define the flow velocity (i.e., the velocity is an $\vec{E} \times \vec{B}$ flow). Even though the model is strictly valid only in a large aspect ratio limit, the equilibrium metric elements, which describe the toroidal geometry, are retained to all orders to allow for poloidal mode coupling.

Single helicity, neoclassical MHD driven tearing modes are demonstrated to exist through numerical simulations. The neoclassical tearing mode is driven by the elimination of the bootstrap current within the island separatrix due to the rapid relaxation of pressure gradients via fast parallel electron heat transport along the closed helical magnetic field lines within the island. However, since the parallel pressure diffusivity is finite, the pressure does not completely equilibrate across the island separatrix. A threshold value for the magnetic perturbation at the mode rational surface is then required to provide a sufficient flattening of the pressure profile about the island to destabilize the island. The scaling of the threshold with the ratio of perpendicular to parallel diffusivities and the local pressure gradient is in qualitative agreement with the analytic predictions. However, the numerically limited $\chi_{\|} / \chi_{\perp}=10^{5}$ is much lower than realistic values.

More recently, the neoclassical enhancement to the ion poloarization current has been predicted to provide a nonlinear island threshold for the formation of magnetic islands. ${ }^{25}$ In this model, the predicted ion polarization induced threshold is on the order of the ion banana width in very collisionless plasmas. The effect of this work is not included in the present simulations. In order to describe this effect, two-fluid equations are needed as well as a very complicated viscosity which would depend upon the mode frequency, nonlinear island width, and the plasma collision frequency.

\section{ACKNOWLEDGMENTS}

The authors gratefully acknowledge Oak Ridge National Labs for access to the FAR code and especially thank Lowell Charlton and Don Spong for numerous conversations on the use and implementation of neoclassical effects in FAR. The cylindrical $\Delta^{\prime}$ values quoted at the end of the fourth paragraph of Section IV were obtained utilizing an adaptation of a delta-prime code developed by Y. Nishimura.

Computer workstations were provided via a grant to the University of Wisconsin from IBM, the DOE Office of Fusion Energy, and the University of Wisconsin, College of Engineering. This work was supported by the United States Department of Energy (DOE) under Grant No. DE-FG02ER53218.

\section{APPENDIX: NEOCLASSICAL CLOSURE}

The starting point for the neoclassical stress-tensor closure used in this model is appropriate in the limit of long collisional mean free path length (or low collision frequency) and accounts for the viscosity between trapped and untrapped particles. ${ }^{20,26-28}$ The stress tensor, $\overrightarrow{\vec{\pi}}$, is represented in a Chew-Goldberger-Low form as

$$
\overrightarrow{\vec{\pi}}_{\alpha} \simeq \overrightarrow{\vec{\pi}}_{\| \alpha}=\left(\frac{\vec{B} \vec{B}}{B^{2}}-\frac{\overrightarrow{\vec{I}}}{3}\right)\left(p_{\|}-p_{\perp}\right)_{\alpha},
$$

where

$$
\left(p_{\|}-p_{\perp}\right)_{\alpha}=-2 m_{\alpha} n_{\alpha} \mu_{\alpha} \frac{\left\langle B^{2}\right\rangle}{\left\langle\left[\left(\vec{B} \cdot \nabla B^{2}\right) / B^{2}\right]^{2}\right\rangle} \frac{\vec{v}_{\alpha} \cdot \nabla B^{2}}{B^{2}} ;
$$

the subscript alpha indicates electrons or ions. The $\langle\Phi\rangle$ is a flux surface average of the quantity $\Phi$. The viscous damping frequencies are approximated by ${ }^{20,27}$

$$
\begin{aligned}
& \mu_{e} \simeq \frac{2.3 \epsilon_{r}^{1 / 2} \nu_{e}}{\left(1+1.07 \nu_{* e}^{1 / 2}+1.02 \nu_{* e}\right)\left(1+1.07 \nu_{e} \epsilon_{r}^{3 / 2}\right)}, \\
& \mu_{i} \simeq \frac{0.66 \epsilon_{r}^{1 / 2} \nu_{i}}{\left(1+1.03 \nu_{* i}^{1 / 2}+0.31 \nu_{* i}\left(1+0.66 \nu_{* i} \epsilon_{r}^{3 / 2}\right)\right.},
\end{aligned}
$$

where 


$$
\nu_{* \alpha}=\nu_{\alpha} \epsilon_{r}^{-3 / 2} R_{0} q V_{T_{\alpha}}^{-1}
$$

in which $\nu_{\alpha}$ is the collision frequency of plasma species $\alpha$ and $V_{T_{\alpha}}$ is the thermal velocity of species $\alpha$. Here, $\epsilon_{r} \equiv \rho / R$ is the ratio of the local plasma minor radius to the plasma major radius. The species velocities which appear are given by

$$
\vec{v}_{e}=\vec{v}-\frac{m_{i}}{e \rho_{D}} \vec{J}
$$

and in the case of ions $\vec{v}_{i}=\vec{v}$. In both Ohm's law and the momentum balance the relevant expression for the viscous force will be

$$
\nabla \cdot \vec{\pi}=\vec{B} \vec{B} \cdot \nabla\left(\frac{f}{B^{2}}\right)+\frac{f}{B^{2}} \nabla\left(\frac{B^{2}}{2}\right)-\frac{f}{B^{2}} \vec{B} \times \nabla \times \vec{B}-\frac{1}{3} \nabla f,
$$

where $f=p_{\|}-p_{\perp}$. In the case of electrons, this form can be reduced to a form which depends on the pressure gradients, ${ }^{12,29}$

$$
\begin{aligned}
-\frac{R^{2}}{I} \vec{B} \cdot \nabla \cdot \vec{\pi}_{e} \simeq & \frac{\partial p_{1}}{\partial \rho}\left\{\frac{-\beta}{\epsilon^{2}} \frac{\mu_{e}}{\nu_{e}} \frac{\left\langle B_{0}^{2}\right\rangle}{\left\langle\left[\left(\vec{B}_{0} \cdot \nabla B_{0}^{2}\right) / B_{0}^{2}\right]^{2}\right\rangle} \frac{1}{R^{2} B_{0}^{4}} \frac{\rho}{q}\left(\frac{1}{\rho} \frac{\partial B_{0}^{2}}{\partial \theta}\right)^{2}\right\} \\
& +\frac{1}{\rho} \frac{\partial p_{1}}{\partial \theta}\left\{\frac{\beta}{\epsilon^{2}} \frac{\mu_{e}}{\nu_{e}} \frac{\left\langle B_{0}^{2}\right\rangle}{\left\langle\left[\left(\vec{B}_{0} \cdot \nabla B_{0}^{2}\right) / B_{0}^{2}\right]^{2}\right\rangle} \frac{1}{R^{2} B_{0}^{4}} \frac{1}{\rho} \frac{\partial B_{0}^{2}}{\partial \theta} \frac{\rho}{q}\left[\frac{\partial B_{0}^{2}}{\partial \rho}-\frac{I}{R^{2}} \frac{d I}{d \rho}\right]\right\} \\
& +\frac{1}{\rho} \frac{\partial p_{1}}{\partial \theta}\left\{\frac{-2 \beta}{\epsilon^{3}} \frac{\mu_{e}}{\nu_{e}} \frac{\left\langle B_{0}^{2}\right\rangle}{\left\langle\left[\left(\vec{B}_{0} \cdot \nabla B_{0}^{2}\right) / B_{0}^{2}\right]^{2}\right\rangle} \frac{1}{R^{4} B_{0}^{4}} \frac{\rho I}{q} \frac{1}{\rho} \frac{\partial B_{0}^{2}}{\partial \theta} \frac{d I}{d \rho}\right\} \\
& +\frac{\partial p_{1}}{\partial \zeta}\left\{\frac{2 \beta}{\epsilon^{3}} \frac{\mu_{e}}{\nu_{e}} \frac{\left\langle B_{0}^{2}\right\rangle}{\left\langle\left[\left(\vec{B}_{0} \cdot \nabla B_{0}^{2}\right) / B_{0}^{2}\right]^{2}\right\rangle} \frac{1}{R^{4} B_{0}^{4}} \frac{1}{\rho} \frac{\partial B_{0}^{2}}{\partial \theta} I \frac{d I}{d \rho}\right\} \\
& +\frac{\partial \psi_{1}}{\partial \rho}\left\{\frac{1}{\epsilon^{2}} \frac{\mu_{e}}{\nu_{e}} \frac{\left\langle B_{0}^{2}\right\rangle}{\left\langle\left[\left(\vec{B}_{0} \cdot \nabla B_{0}^{2}\right) / B_{0}^{2}\right]^{2}\right\rangle} \frac{1}{R^{4} B_{0}^{4}} \frac{d I}{d \rho}\left(\frac{1}{\rho} \frac{\partial B_{0}^{2}}{\partial \theta}\right)^{2}\right\} \\
& +\frac{1}{\rho} \frac{\partial \psi_{1}}{\partial \theta}\left\{\frac{-1}{\epsilon^{2}} \frac{\mu_{e}}{\nu_{e}} \frac{\left\langle B_{0}^{2}\right\rangle}{\left\langle\left[\left(\vec{B}_{0} \cdot \nabla B_{0}^{2}\right) / B_{0}^{2}\right]^{2}\right\rangle} \frac{1}{R^{4} B_{0}^{4}} \frac{d I}{d \rho} \frac{\partial B_{0}^{2}}{\partial \rho} \frac{1}{\rho} \frac{\partial B_{0}^{2}}{\partial \theta}\right\} .
\end{aligned}
$$

An additional approximation is made that the viscosity coefficients are assumed to be constant across the plasma extent. In the vicinity of an island this is probably a reasonable approximation.

The neoclassical contribution to the stress tensor term in the vorticity evolution equation is analogously derived from the above expressions. The form which has been used in the simulations is given by

$$
\begin{aligned}
\nabla \zeta \cdot \nabla \times \nabla \cdot \vec{\pi}= & \left\{\frac{\partial^{2} \phi_{1}}{\partial \rho^{2}}\right\}\left\{\frac{1}{\epsilon^{2}} \frac{\mu_{i}}{\nu_{e i}} \frac{1}{B^{2} R^{2}} \frac{1}{R^{2}} \frac{\left\langle B^{2}\right\rangle}{\left\langle\left[\left(\vec{B} \cdot \nabla B^{2}\right) / B^{2}\right]^{2}\right\rangle}\left(\frac{1}{\rho} \frac{\partial B^{2}}{\partial \theta}\right)^{2}\right\} \\
& +\left\{\frac{\partial \phi_{1}}{\partial \rho}\right\}\left\{-\frac{1}{\epsilon^{2}} \frac{\mu_{i}}{\nu_{e i}} \frac{1}{B^{2} R^{2}} \frac{1}{\rho} \frac{\partial^{2}}{\partial \rho \partial \theta}\left[\frac{B^{2}}{R^{2}} \frac{\left\langle B^{2}\right\rangle}{\left\langle\left[\left(\vec{B} \cdot \nabla B^{2}\right) / B^{2}\right]^{2}\right\rangle} \frac{1}{\rho} \frac{\left.\partial B^{2}\right]}{\partial \theta}\right]\right\} \\
& +\left\{\frac{1}{\rho^{2}} \frac{\partial^{2} \phi_{1}}{\partial \theta^{2}}\right\}\left\{\frac{1}{\epsilon^{2}} \frac{\mu_{i}}{\nu_{e i}} \frac{1}{B^{2} R^{2}} \frac{1}{R^{2}} \frac{\left\langle B^{2}\right\rangle}{\left\langle\left[\left(\vec{B} \cdot \nabla B^{2}\right) / B^{2}\right]^{2}\right\rangle}\left(\frac{\partial B^{2}}{\partial \rho}\right)^{2}\right\} \\
& +\left\{\frac{1}{\rho} \frac{\partial \phi_{1}}{\partial \theta}\right\}\left\{\frac{1}{\epsilon^{2}} \frac{\mu_{i}}{\nu_{e i}} \frac{1}{B^{2} R^{2}} \frac{\partial^{2}}{\partial \rho \partial \theta}\left[\frac{B^{2}}{R^{2}} \frac{\left\langle B^{2}\right\rangle}{\left\langle\left[\left(\vec{B} \cdot \nabla B^{2}\right) / B^{2}\right]^{2}\right\rangle} \frac{1}{\rho} \frac{\partial B^{2}}{\partial \rho}\right]\right\} \\
& +\left\{\frac{1}{\rho} \frac{\partial^{2} \phi_{1}}{\partial \rho \partial \theta}\right\}\left\{-\frac{2}{\epsilon^{2}} \frac{\mu_{i}}{\nu_{e i}} \frac{1}{B^{2} R^{2}} \frac{1}{R^{2}} \frac{\left\langle B^{2}\right\rangle}{\left\langle\left[\left(\vec{B} \cdot \nabla B^{2}\right) / B^{2}\right]^{2}\right\rangle} \frac{\partial B^{2}}{\partial \rho} \frac{1}{\rho} \frac{\partial B^{2}}{\partial \theta}\right\} .
\end{aligned}
$$


${ }^{1}$ Z. Chang, E.D. Fredrickson, J.D. Callen, K.M. McGuire, M.G. Bell, R.V. Budny, C.E. Bush, D.S. Darrow, A.C. Janos, L.C. Johnson, H.K. Park, S.D. Scott, J.D. Strachan, E.J. Synakowski, G. Taylor, R.M. Wieland, M.C. Zarnstorff, S.J. Zweben, and the TFTR team, Nucl. Fusion 34, 1309 (1994).

${ }^{2}$ Z. Chang, J.D. Callen, E.D. Fredrickson, R.V. Budny, C.C. Hegna, K.M. McGuire, M.C. Zarnstorff, and the TFTR team, Phys. Rev. Lett. 74, 4663 (1995).

${ }^{3}$ E. Fredrickson, S. Batha, M.G. Bell, R.V. Budny, C.E. Bush, Z. Chang, C.Z. Cheung, D.S. Darrow, J. Dunlap, G.Y. Fu, H.W. Herrmann, H. Hsuan, R. Majeski, D.K. Mansfield, E. Mazzucato, K.M. McGuire, D.R. Mikkelsen, M. Murakimi, R. Nazikan, A.C. Janos, C.K. Philips, S.A. Sabbagh, G.L. Schmidt, S.D. Scott, J.D. Strachan, E.J. Synakowski, H. Takahashi, G. Taylor, J.R. Wilson, K.L. Wong, M.C. Zarnstorff, S.J. Zweben, and the TFTR team, in Plasma Physics and Controlled Nuclear Fusion Research 1994 (International Atomic Energy Agency, Vienna, 1994).

${ }^{4}$ B.A. Carreras, B.V. Waddell, H.R. Hicks, and S.J. Lynch, Phys. Rev. A 18, 2732 (1978).

${ }^{5}$ See National Technical Information Service Document No. DE6008946 [W.X. Qu and J.D. Callen, "Nonlinear growth of a single neoclassical MHD tearing mode in a tokamak," U.W. Plasma Report 85-5 (1985)]. Copies may be ordered from the National Technical Information Service, Springfield, VA 22161.

${ }^{6}$ R. Carrera, R.D. Hazeltine, and M. Kotschenreuther, Phys. Fluids 29, 899 (1986).

${ }^{7}$ C.C. Hegna and J.D. Callen, Phys. Fluids B 4, 1855 (1992).

${ }^{8}$ P. H. Rutherford, Phys. Fluids 16, 1903 (1973).

${ }^{9}$ R. Fitzpatrick, Phys. Plasmas 2, 825 (1995). L. E. Zahakarov (private communication).

${ }^{10}$ H.P. Furth, J. Killeen, and M.N. Rosenbluth, Phys. Fluids 6, 459 (1963).

${ }^{11}$ J.D. Strachan, M.Bitter, A.T. Ramsey, M.C. Zarnstorff, V. Arunasalam, M.G. Bell, N.L. Bretz, R. Budny, C.E. Bush, S.L. Davis, H.F. Dylla, P.C. Efthimion, R.J. Fonck, E. Fredrickson, H.P. Furth, R.J. Goldston, L.R. Grisham, B. Grek, R.J. Hawryluk, W.W. Heidbrink, H.W. Hendel, K.W. Hill, H. Hsuan, K.P. Jaehnig, D.L. Jassby, F. Jobes, D.W. Johnson, L.C. Johnson, R. Kaita, J. Kamperschroer, R.J. Knize, T. Kozub, H. Kugel, B. LeBlanc, F. Levinton, P.H. La Marche, D.M. Manos, D.K. Mansfield, K.
McGuire, D.H. McNeill, D.M. Meade, S.S. Medley, W. Morris, D. Mueller, E.B. Nieschmidt, D.K. Owens, H. Park, J. Schivell, G. Schilling, G.L. Schmidt, S.D. Scott, S. Sesnic, J.C. Sinnis, F.J. Stauffer, B.C. Stratton, G.D. Strait, G. Taylor, H.H. Towner, M. Ulrickson, S. von Goeler, R. Wieland, M.D. Williams, K.L. Wong, S. Yoshikawa, K.M. Young, and S.J. Zweben, Phys. Rev. Lett. 58, 1004 (1987).

${ }^{12}$ T.A. Gianakon, Ph.D. thesis, Univeristy of Wisconsin-Madison, 1996 (T.A. Gianakon, UW-CPTC 96-1).

${ }^{13}$ J.A. Holmes, J. Comput. Phys. 36, 35 (1980).

${ }^{14}$ V.E. Lynch, B.A. Carreras, H.R. Hicks, J.A. Holmes, and L. Garcia, Comput. Phys. Commun. 24, 465 (1981).

${ }^{15}$ J.A. Holmes, B.A. Carreras, H.R. Hicks, V.E. Lynch, and K.E. Rothe, Phys. Fluids 25, 800 (1982).

${ }^{16}$ L.A. Charlton, J.A. Holmes, H.R. Hicks, V.E. Lynch, and B.A. Carreras, J. Comput. Phys. 63, 107 (1986)

${ }^{17}$ W.D. D'haeseller, W.N.G. Hitchon, J.D. Callen, and J.L. Shohet, Flux Coordinates and Magnetic Field Structure (Springer-Verlag, New York, 1991).

${ }^{18}$ H.R. Strauss, Nucl. Fusion 23, 649 (1983).

${ }^{19}$ R.D. Hazeltine and J.D. Meiss, Phys. Rep. 121, 1 (1985).

${ }^{20}$ J.D. Callen, W.X. Qu, K.D. Siebert, B.A. Carreras, K.C. Shaing, and D.A. Spong, in Plasma Physics and Controlled Nuclear Fusion Research 1986 (International Atomic Energy Agency, Vienna, 1987), Vol. I, p. 157.

${ }^{21}$ A.H. Glasser, J.M. Greene, and J.L. Johnson, Phys. Fluids 18, 875 (1975).

${ }^{22}$ M. Kotschenreuther, R.D. Hazeltine, and P.J. Morrison, Phys. Fluids 28, 294 (1985).

${ }^{23}$ C.C. Hegna and J.D. Callen, Phys. Fluids B 4, 4072 (1992).

${ }^{24}$ Z. Chang and J.D. Callen, Nucl. Fusion 30, 219 (1990).

${ }^{25}$ H.R. Wilson, J.W. Connor, R.J. Hastie, and C.C. Hegna, Phys. Plasmas 3, 248 (1996).

${ }^{26}$ J.P. Wang and J.D. Callen, Phys. Plasmas B 5, 3207 (1993).

${ }^{27}$ F.L. Hinton and R.D. Hazeltine, Rev. Mod. Phys. 48, 239 (1976).

${ }^{28}$ S.P. Hirshman and D.J. Sigmar, Nucl. Fusion 9, 1079 (1981).

${ }^{29}$ D.A. Spong, K.C. Shaing, B.A. Carreras, J.D. Callen, and L. Garcia (private communication, available as "Transition from resistive ballooning to neoclassical magnetohydrodynamic pressure-gradient-driven instability,", Paper No. ORNL/TM-10947, 1988). 